\title{
Bacteraemia Variation During The COVID-19 Pandemic; A Multi-Centre UK Secondary Care Ecological Analysis.
}

\author{
Sarah Denny \\ Chelsea and Westminster Hospital NHS Foundation Trust \\ Timothy M Rawson \\ Imperial College Healthcare NHS Trust \\ Giovanni Satta \\ Imperial College Healthcare NHS Trust \\ Scott JC Pallett \\ Royal Centre for Defence Medicine \\ Ahmed Abdulaal \\ Chelsea and Westminster Hospital NHS Foundation Trust \\ Stephen Hughes \\ Chelsea and Westminster Hospital NHS Foundation Trust \\ Mark Gilchrist \\ Imperial College Healthcare NHS Trust \\ Nabeela Mughal \\ Chelsea and Westminster Hospital NHS Foundation Trust \\ Luke S P Moore ( $D$ l.moore@imperial.ac.uk) \\ Imperial College London https://orcid.org/0000-0001-7095-7922
}

\section{Research article}

Keywords: Microbiology, Blood culture, Coronavirus, SARS-CoV-2

Posted Date: December 1st, 2020

DOI: https://doi.org/10.21203/rs.3.rs-113509/v1

License: (a) (i) This work is licensed under a Creative Commons Attribution 4.0 International License. Read Full License 


\section{Abstract}

Objectives - We investigated for change in blood stream infections (BSI) with Enterobacterales, coagulase negative staphylococci (CoNS), Streptococcus pneumoniae, and Staphylococcus aureus during the first UK wave of SARS-CoV-2 across six London hospitals.

Methods - A retrospective multicentre ecological analysis was undertaken evaluating all blood cultures taken from adults from 01 April 2017 to 30 April 2020 across six acute hospitals in London. Linear trend analysis and ARIMA models allowing for seasonality were used to look for significant variation.

Results -119,584 blood cultures were included. At the height of the UK SARS-CoV-2 first wave in April 2020, Enterobacterales bacteraemias were at an historic low across two London trusts (63/3814, 1.65\%), whilst CoNS were at an historic high (173/3814, 4.25\%). This differed significantly for both Enterobacterales $(\mathrm{p}=0.013)$ and CoNS $(\mathrm{p}<0.01)$, when compared with prior periods, even allowing for seasonal variation. $S$. pneumoniae $(\mathrm{p}=0.631)$ and $S$. aureus $(\mathrm{p}=0.617) \mathrm{BSI}$ did not vary significant throughout the study period.

Conclusions - Significantly fewer than expected Enterobacteriales BSI occurred during the UK peak of the COVID-19 pandemic; identifying potential causes, including potential unintended consequences of national self-isolation public health messaging, is essential. High rates of CoNS BSI, presumably representing contamination associated with increased use of personal protective equipment, may result in inappropriate antimicrobial use and indicates a clear area for intervention during further waves.

\section{Introduction}

Severe Acute Respiratory Syndrome coronavirus 2 (SARS-CoV-2) began in December 2019 in China. Transmission within Europe, including the United Kingdom (UK), was confirmed by the end of February 2020. In the UK, social distancing and self-quarantine measures were subsequently implemented, aimed at slowing transmission. Furthermore, in an attempt to prevent overwhelming hospital capacity, those with high temperature and/or new continuous cough (i.e. symptoms consistent with COVID-19, but also other infections) were advised to stay home and seek advice online or via a national telephone service (1). Elective clinical services were also reduced, with cancellation of non-urgent clinics and surgery. Extensive self-isolation practices and decreased utilisation of healthcare services has potentially impacted on direct care for both COVID-19 and non-COVID-19 clinical presentations.

Altered presentation of bloodstream infections (BSI) to healthcare is one potential indirect consequence of national public health measures. Whilst some organisms associated with BSIs have a clear communicable component (for example Streptococcus pneumoniae, Neisseria meningitidis and Staphylococcus aureus) (2-4), others are likely to arise endogenously (5). Therefore, the incidence of communicable pathogens may decrease secondary to social distancing, or increase due to increased time spent with household contacts, while incidence of likely endogenous pathogens, such as Escherichia coli and other Enterobacterales, should be less affected. Potential changes must be considered however in the context of the seasonality for Enterobacteriales (peaks seen in summer) $(6,7)$ and S. pneumoniae (peaks in winter). Finally, coagulase negative staphylococci (CoNS) are skin commensals and frequent contaminants of bloods cultures. Lower rates are seen with increased phlebotomy expertise/appropriate skin decontamination. International targets for contamination are less than 3\% (8). It may be hypothesised that incidence would increase with changes to working conditions, including implementation of personal protective equipment (PPE) for staff.

With NHS England data suggesting that emergency presentations to secondary care were down 29.4\% in March 2020 compared to March 2019 (9), there are concerns over delayed or missed presentations with non-COVID-19 infections. To explore this, we investigated the incidence of BSIs during the initial months of the COVID-19 pandemic, focusing on Enterobacterales and CoNS, against variations in rates across the preceding three years. We used Enterobacteriales, S. pneumoniae, and S. aureus BSI as indicator organisms for missed bacteraemia presentations and CoNS BSIs as a surrogate for contamination.

\section{Methods}

Study setting and design

A retrospective multicentre ecological analysis was undertaken evaluating all blood culture (BC) samples from adults (aged 17 years and above) over a three-year period from 01 April 2017 to 30 April 2020 across six acute hospitals in London, serving a population approximating three million.

A hub-and-spoke laboratory network with a centralized microbiology laboratory processes samples from multiple hospitals in accordance with UK laboratory standard operating procedures (10) with minor local variation. BCs were collected at each hospital, without pre-incubation, and transported to the centralized laboratory. They were subsequently incubated using a BACTEC system (Becton Dickinson, Franklin Lakes, NJ, USA). Organisms were identified by matrix assisted laser desorption/ionisation-time-of-flight (MALDI-TOF) mass spectroscopy (Bruker Daltonik GmbH, Bremen, Germany). Susceptibility testing was undertaken using disk diffusion using European Committee on Antimicrobial Susceptibility testing methods and interpretative criteria (11).

\section{Data collection}

Microbiological data was extracted from Sunquest Laboratory V8.3 (Tucson, AZ, USA). For the purposes of this study, Enterobacterales included Escherichia coli, Klebsiella spp., Serratia spp., Enterobacter spp., Proteus spp., Citrobacter spp., Hafnia spp., Morganella spp., and Pantoea spp. All CoNS, S.pneumoniae and S.aureus isolated from BCs were included; the seasonality of the latter two has been extensively described (12-15). If more than one 
pathogen was isolated from a BC, each was recorded individually at either genus or species level. Samples with multiple Enterobacterales or CoNS were recorded as a single positive BC. Repeated positives within a 14-day period were not de-duplicated and patient records were not assessed to determine if CoNS BSIs represented true infection.

Data analysis

To explore seasonal variations in BSI rates, results were classified as spring (March-May), summer (June-August), autumn (September-November) and winter (December-February). For spring 2017, only April and May data were available. March and April 2020 were considered as the period when social distancing measures were in place, encompassing the UK COVID-19 peak (16). The same period in 2019 was analysed for comparison.

To explore changing trends in BCs results during COVID-19, two different statistical models were used; both (first) allowing for seasonality, and (second) then separately obviating any potential seasonality. The first method was a time-series analysis performed in R (R Core Team). After testing for the absence of first-order autocorrelations with the Durbin-Watson statistic, univariable autoregressive integrated moving average (ARIMA) models were fitted to data from CoNS, Enterobacterales, S.pneumoniae, and S.aureus from April 2017 to December 2019. These models were used to forecast estimated BSI rates for January to June 2020. To explore differences between observed and expected BSI rates during the COVID-19 peak, they were graphically compared.

The second method was a linear trend analysis of variations in observed rates of BSIs. Z-scores were calculated for Enterobacterales, CoNS, S.penumoniae and S.aureus using SPSS (IBM Corp, Armonk, NY, USA). Data were checked for normality using the Shapiro-Wilk test. Where non-normal distribution was identified, data were transformed prior to analysis. Z-scores were then calculated each month by applying the standard formula $z=(x-\mu) /$ $\sigma(x=$ raw score $\mu=$ mean, $\sigma=$ standard deviation). Statistical significance for $z$-scores were determined using $R$ (R Core Team).

\section{Study approval}

This analysis was registered with North West London Pathology hosted by Imperial College Healthcare NHS Trust as a service evaluation (reference PAT_012) to investigate BC contamination rates. Individual consent was not indicated for this ecological level analysis reporting only aggregated data.

\section{Results}

During April 2017 to April 2020, 133,856 BCs were identified. Of those, 12,625 were from an external source (non-networked hospital), 2,002 unclassifiable, and 729 not processed, leaving 119,584 for analysis. The overall number of BC requests remained stable throughout the entire period in terms of absolute numbers. From the 119,584 BCs, no pathogen/microorganism was detected in 109,144 (91.27\%). Growth was demonstrated in 10,440 cultures (8.73\%). Enterobacterales were the most commonly isolated pathogen (3,508 cultures, $2.93 \%$ of total), followed by CoNS (2,855 cultures, $2.39 \%)$. Full details of cultured microorganisms and number of tests are provided (Table 1).

\section{Seasonal trends}

Seasonal trends were observed for S.pneumoniae and Enterobacterales. The seasonality of S.pneumoniae (preponderance towards winter months) is shown in Figure 1D, whilst the seasonality of Enterobacterales (peaks in summer months) is shown in Figure 1B. Across the cohort, Enterobacterales as a percentage of all BCs taken was lowest in the winter (2.49\% 2017, 2.54\% 2018 and $2.63 \%$ 2019), with a general trend towards sequentially rising throughout spring and into summer before peaking in autumn (3.42 \% 2017, 3.56\% 2018 and 3.22\% 2019) (Figure 2). For CoNS the mean percentage over the study period is $2.39 \%$. There was little seasonal variation prior to COVID-19 (Figure 2). For S. aureus the linear mean over the study period was 27.57 , with significant variation over time, but little evidence of recursive seasonality (Figure 1C).

\section{Bacteraemia variation during the UK COVID-19 peak March and April 2020}

In April 2020, Enterobacterales as a percentage of all BCs taken were at their lowest during the study period at $1.65 \%$ (second lowest March 2020 at $1.71 \%$; Figure 2). In contrast, CoNS, as a percentage of all BCs taken, were at their highest during the study period at $4.25 \%$ (second highest in March 2020 at $4.08 \%$ ) (Figure 2).

The linear trend analysis (Table 2) for Enterobacterales, CoNS, S.aureus and S.pneumoniae from April 2017 to April 2020 suggests significant differences in BSI rates, particularly during the COVID-19 period. Enterobacterales BSIs were significantly lower than the mean in February, March, and April 2020 only (Z-scores; -2.57, -2.48, and 2.48, respectively; $\mathrm{p}<0.05$ ). In contrast, CoNS BSIs were significantly above the mean for March and April 2020 (Z-scores; 2.77 , 3.61 respectively; $p$ < 0.01). Numbers detected did not deviate significantly from the mean from April 2017 until February and March 2020 , respectively for these organisms. S.aureus BSIs did not vary significantly from mean throughout the period analysed. S.pneumoniae BSIs did not vary significantly from the mean throughout the period analysed, except for in December 2019.

The ARIMA model, constructed to allow for any seasonality, is represented in Figure 3A-D and demonstrates historic observed BSI (April 2017-December 2019) and then predicted versus observed BSI from January 2020 to June 2020 . Once seasonality is allowed for, this also showed a significant divergence in observed versus predicted Enterobacterales and CoNS from February to April 2020. Fewer numbers of Enterobacteriales BSIs and higher numbers of CoNS BSIs were seen. Numbers of S.aureus and S.pneumoniae BSI remained within the limits of confidence of predicted based on historical trends. 


\section{Discussion}

In our multi-centre BSI ecological analysis we find during the period of COVID-19 social distancing and self-isolation a significant reduction in Enterobacteriales BSIs verified across two different statistical models. Similarly, there was a significant increase in CoNS (presumptively contamination) BSI, whilst BSI with ostensibly communicable organisms (S.aureus and S.pneumoniae) remained stable. The observation of reduced Enterobacterales BSI immediately preceding and contemporaneous with public health measures, at a time when they would usually be increasing in incidence, requires urgent consideration $(6,7)$. Given that Enterobacterales BSI could be considered non-communicable and likely endogenous in origin, incidence would be expected to remain similar allowing for seasonality. This observed reduction may reflect changes in presentation behaviour, with patients either selfisolating with 'COVID-19 symptoms' (e.g. fever), or avoiding healthcare due to fear of COVID-19 exposure. Certainly, emergency department attendances were lower than expected (9), in keeping with patients not presenting. Figures from England in 2018/19 show a 30-day mortality rate of 10.4 per 100,000 of the population and case fatality rate of $13.8 \%$ of cases (17) for Enterobacteriales BSIs and there is concern that patients experiencing but not presenting with a BSI may have suffered fatalities in the community.

Widespread cancellation of elective surgical procedures offers an alternative explanation for the reduction in Enterobacteriales BSIs. Gastrointestinal (GI) and urological procedures are a well-established source of Enterobacterales BSI $(18,19)$. Furthermore, many patients presenting to hospital who fit the broad case definition for COVID-19 are rapidly started on broad-spectrum antimicrobials. As antimicrobials affect the detection rate of Enterobacterales from BCs (20), this may also be impacting our observed case rate.

The increased number of CoNS BSIs seen during the pandemic may reflect increased rates of contamination. Contamination rates are reduced with increased phlebotomy expertise, but with staff performing procedures in unfamiliar PPE, the practicalities of venepuncture might prove more difficult. Additionally, more BCs are likely to be taken in patients with COVID-19 due to its febrile nature. This has been substantiated by Sepulveda et al. (21) who found a surge in BCs during March 2020, the majority of which were for SARS-CoV-2 positive patients, including repeated sampling. They found that CoNS accounted for $59.7 \%$ of positive BCs in these patients. Furthermore, data from Hughes et al (22) has demonstrated infrequent confirmed secondary bacterial infection, approximately 3\%-6\%. When planning for future COVID-19 peaks and/or other pandemics, these findings would support the need for dedicated teaching on aseptic non-touch techniques (ANTT) whilst in PPE, in an attempt to reduce contamination.

This could also potentially impact on antimicrobial stewardship and resistance (23). Infection teams may recommend the addition of glycopeptides when Gram-positive cocci (GPC) are isolated from BCs, in the presence of a central line, whilst waiting full identification and sensitivities. To prevent unnecessary glycopeptide prescriptions, potential adverse drug events, antimicrobial resistance, and increased costs, it is important to have COVID-19 specific antimicrobial guidance reflecting the likelihood of increased $\mathrm{BC}$ contamination.

Our study has several limitations. We looked at total number of blood culture requests and so repeated samples from the same patients may have been included. Also, repeated positive BCs within a 14-day period were not de-duplicated, which may have affected incidence. Furthermore, timing or setting of sampling (e.g. ED versus inpatient) was not assessed and thus whether CoNS BSIs were contaminants or true BSIs and whether Enterobacteriales BSIs were community or hospital acquired. Given the increased number of intensive care admissions with COVID-19 during this period, it might be that the rate of CoNS BSIs would increase due to more patients having central venous access. To gain finer resolution on the causes for the observed fall Enterobacterales BSI, it would be necessary to look at community and hospital acquired BSI related to elective GI/urological procedures over the same time frame. In addition, because this was an ecological analysis, we did not describe the epidemiology of patients including whether they had tested positive for COVID-19 or not, nor did we use a COVID-free hospital as a control.

\section{Conclusion}

During the peak of the COVID-19 pandemic in London, significantly fewer Enterobacterales BSI occurred, alongside fewer documented emergency presentations. This potentially reflects reduced presentations of Enterobacterales BSI due to patients not presenting to secondary care. We suggest more nuanced public health messaging around self-isolation for febrile illnesses to ensure patients present to healthcare where necessary. High rates of CoNS BSI during the COVID-19 peak, potentially due to contamination, might reflect an unintended consequence of PPE. We suggest increased training for ANTT procedures whilst using PPE to prepare for a second CoVID-19 wave or any future pandemics. We suggest specific antimicrobial guidelines for patients with COVID-19, not only accounting for fine resolution epidemiology on the frequency of bacterial co-infection, but also reflecting caution in reacting to GPCs in $\mathrm{BC}$ bottles.

\section{Abbreviations}

CoNS - coagulase negative staphylococci

S.pneumo - Streptococcus pneumoniae

GNR-E - Gram Negative Rods - Enterobacterales

GNR-NE - Gram Negative Rods- Non Enterobacterales

Other - including Group A/B/C/G Streptococci, Gram positive rods, Actinobacillus spp., Aerococcus spp., Microbacterium spp., Micrococcus spp 


\section{Declarations}

\section{Ethics approval}

This analysis was registered with North West London Pathology hosted by Imperial College Healthcare NHS Trust as a service evaluation (reference PAT_012) to investigate BC contamination rates. Ethics approval and individual consent was not indicated for this ecological level analysis reporting only aggregated data.

\section{Acknowledgements}

LSPM and TMR acknowledge support from the National Institute of Health Research (NIHR) Imperial Biomedical Research Centre (BRC) and the National Institute for Health Research Health Protection Research Unit (HPRU) in Healthcare Associated Infection and Antimicrobial Resistance at Imperial College London in partnership with Public Health England.

Dr Pater Hart for helping to clean the data and perform statistical analyses.

The views expressed in this publication are those of the authors and not necessarily those of the NHS, the National Institute for Health Research, or the UK Department of Health.

\section{Authors' contributions}

SD, GS and LSPM designed the study methodology. SD collated the data. All authors reviewed the results, SD, TMR and GS lead the data analysis, with all authors reviewing the resulting themes and contributed comments. SD drafted the initial manuscript with all authors contributing significantly to revising this for submission. All authors agreed on the final version for submission to the journal.

\section{Funding}

This research did not receive any grant from funding agencies in the public or commercial sectors.

\section{Potential conflicts of interests}

LSPM has consulted for DNAelectronics (2015-18), Dairy Crest (2017-2018), Umovis Lab (2020), bioMerieux (2013-2020), received speaker fees from Profile Pharma (2018) and Pfizer (2018-2020), received research grants from the National Institute for Health Research (2013-2020), CW+ Charity (20182020), and Leo Pharma (2016), and received educational support from Eumedica (2016-2018).

GS is currently supported by a Clinical Academic Research Partnership awarded by the Medical Research Council. SJCP has received a research grant from the Scientific Exploration Society. NM has received speaker fees from Beyer (2016) and Pfizer (2019) and received educational support from Eumedica (2016) and Baxter (2017).

All other authors have no conflicts of interest to declare.

\section{Consent for publication}

All authors consent to publication of this manuscript in this journal. This work has not been previously published in any other journal.

\section{Availability of data and materials}

The data analysed during the current study and further details on the assays are available from the corresponding author (SD; sarahdenny1@nhs.net) on reasonable request, as long as this meets local ethical and research governance criteria.

\section{References}

1. website N. Check if you or your child has coronavirus symptoms 2020 [Available from: https://www.nhs.uk/conditions/coronavirus-covid19/symptoms/.

2. Weiser JN, Ferreira DM, Paton JC. Streptococcus pneumoniae: transmission, colonization and invasion. Nature reviews Microbiology. 2018;16(6):35567.

3. Rouphael NG, Stephens DS. Neisseria meningitidis: biology, microbiology, and epidemiology. Methods in molecular biology (Clifton, NJ). 2012;799:120.

4. Knox J, Uhlemann A-C, Lowy FD. Staphylococcus aureus infections: transmission within households and the community. Trends in microbiology. 2015;23(7):437-44.

5. Oliveira J, Reygaert WC. Gram Negative Bacteria. 2019. 
6. Al-Hasan MN, Lahr BD, Eckel-Passow JE, Baddour LM. Seasonal Variation in Escherichia coli Bloodstream Infection: A Population-Based Study. Clin Microbiol Infect. 2009;15(10):947-50.

7. Wilson J, Elgohari S, Livermore DM, Cookson B, Johnson A, Lamagni T, et al. Trends among pathogens reported as causing bacteraemia in England, 2004-2008. Clinical Microbiology and Infection. 2011;17(3):451-8.

8. Hall KK, Lyman JA. Updated review of blood culture contamination. Clinical microbiology reviews. 2006;19(4):788-802.

9. England N. A\&E Attendances and Emergency Admissions 2020 [Available from: https://www.england.nhs.uk/statistics/statistical-work-areas/aewaiting-times-and-activity/.

10. England PH. UK SMI B 37: investigation of blood cultures (for organisms other than Mycobacterium species) 2019 [Available from: https://www.gov.uk/government/publications/smi-b-37-investigation-of-blood-cultures-for-organisms-other-than-mycobacterium-species.

11. EUCAST. Clinical breakpoints - breakpoints and guidance 2020 [Available from: https://www.eucast.org/clinical_breakpoints/.

12. Dowell SF, Whitney CG, Wright C, Rose CE, Jr., Schuchat A. Seasonal patterns of invasive pneumococcal disease. Emerging infectious diseases. 2003;9(5):573-9.

13. Numminen E, Chewapreecha C, Turner C, Goldblatt D, Nosten F, Bentley SD, et al. Climate induces seasonality in pneumococcal transmission. Scientific Reports. 2015;5(1):11344.

14. Weinberger DM, Harboe ZB, Viboud C, Krause TG, Miller M, Mølbak K, et al. Pneumococcal disease seasonality: incidence, severity and the role of influenza activity. European Respiratory Journal. 2014;43(3):833.

15. Leekha S, Diekema DJ, Perencevich EN. Seasonality of staphylococcal infections. Clin Microbiol Infect. 2012;18(10):927-33.

16. LSHTM. Peak in COVID-19 deaths occurring in English hospitals passed on 8 April 2020 [Available from: https://www.Ishtm.ac.uk/newsevents/news/2020/peak-covid-19-deaths-occurring-english-hospitals-passed-8-april.

17. England PH. Thirty-day all-cause mortality following MRSA, MSSA and Gram-negative bacteraemia and C. difficile infections 2019 [Available from: https://assets.publishing.service.gov.uk/government/uploads/system/uploads/attachment_data/file/850521/hcai_fatality_report_201819.pdf.

18. Alqarni A, Kantor E, Grall N, Tanaka S, Zappella N, Godement M, et al. Clinical characteristics and prognosis of bacteraemia during postoperative intra-abdominal infections. Crit Care. 2018;22(1):175.

19. Olson ES, Cookson BD. Do antimicrobials have a role in preventing septicaemia following instrumentation of the urinary tract? J Hosp Infect. 2000;45(2):85-97.

20. Scheer CS, Fuchs C, Gründling M, Vollmer M, Bast J, Bohnert JA, et al. Impact of antibiotic administration on blood culture positivity at the beginning of sepsis: a prospective clinical cohort study. Clin Microbiol Infect. 2019;25(3):326-31.

21. Sepulveda J, Westblade LF, Whittier S, Satlin MJ, Greendyke WG, Aaron JG, et al. Bacteremia and Blood Culture Utilization During CoVID-19 Surge in New York City. Journal of Clinical Microbiology. 2020:JCM.00875-20.

22. Hughes S, Troise O, Donaldson H, Mughal N, Moore LSP. Bacterial and fungal coinfection among hospitalised patients with COVID-19: A retrospective cohort study in a UK secondary care setting. Clin Microbiol Infect. 2020; S1198-743X(20)30369-4. doi: 10.1016/j.cmi.2020.06.025. Online ahead of print.

23. Rawson TM, Moore LSP, Castro-Sanchez E, Charani E, Davies F, Satta G, et al. COVID-19 and the potential long-term impact on antimicrobial resistance. Journal of Antimicrobial Chemotherapy. 2020;75(7):1681-4.

\section{Tables}

Table 1: Summary of positive and negative blood cultures collected during the period April 2017 to April 2020 across a six London hospital network.

\begin{tabular}{|llll|}
\hline Blood cultures with no growth (N; \% of all blood cultures) & Group & Number of blood cultures & \% of total blood cultures \\
\hline Blood cultures with isolate identified (N; \% of all blood cultures) & & 109,144 & $91.27 \%$ \\
\cline { 2 - 4 } & CoNS & 2,855 & $2.39 \%$ \\
\hline & S.pneumo & 78 & $0.07 \%$ \\
\hline GNR-E & 3,508 & $2.93 \%$ \\
\hline GNR-NE & 790 & $0.66 \%$ \\
\hline Other & 1975 & $1.65 \%$ \\
\hline S.aureus & 988 & $0.83 \%$ \\
\hline
\end{tabular}

Table 2: Variation in blood stream infection during the period April 2017 to April 2020 across a six London hospital network for Enterobaterales, CoNS, S.aureus and S.pneumoniae. Z-score calculated as variation from linear mean. The month of March and April 2020 during the COVID-19 social isolation 
measures are highlighted in grey.

Page $7 / 12$ 


\begin{tabular}{|c|c|c|c|c|c|c|c|c|}
\hline Month & $\begin{array}{l}\text { Coagulase negative } \\
\text { staphylococcus Z-score. }\end{array}$ & $\begin{array}{l}p- \\
\text { value }\end{array}$ & $\begin{array}{l}\text { Enterobacterales } \\
\text { Z-score }\end{array}$ & $\begin{array}{l}p- \\
\text { value }\end{array}$ & $\begin{array}{l}\text { Staphylococcus } \\
\text { aureus Z-score }\end{array}$ & $\begin{array}{l}p- \\
\text { value }\end{array}$ & $\begin{array}{l}\text { Streptococcus } \\
\text { pneumoniae Z-score }\end{array}$ & $\begin{array}{l}p- \\
\text { value }\end{array}$ \\
\hline Apr-17 & -0.908 & 0.364 & 0.493 & 0.622 & 0.341 & 0.733 & -0.480 & 0.613 \\
\hline $\begin{array}{l}\text { May- } \\
17\end{array}$ & 0.103 & 0.918 & 0.330 & 0.742 & 0.061 & 0.952 & -0.480 & 0.631 \\
\hline $\begin{array}{l}\text { Jun- } \\
17\end{array}$ & 0.215 & 0.830 & 0.161 & 0.872 & -0.501 & 0.617 & -0.914 & 0.361 \\
\hline Jul-17 & -0.437 & 0.662 & 0.045 & 0.964 & 0.061 & 0.952 & -0.047 & 0.963 \\
\hline $\begin{array}{l}\text { Aug- } \\
17\end{array}$ & 0.269 & 0.788 & 1.343 & 0.179 & 0.341 & 0.733 & -0.914 & 0.361 \\
\hline $\begin{array}{l}\text { Sep- } \\
17\end{array}$ & -0.069 & 0.945 & 0.653 & 0.514 & 0.201 & 0.841 & -0.914 & 0.361 \\
\hline Oct-17 & 0.103 & 0.918 & 0.808 & 0.419 & 0.061 & 0.952 & -0.914 & 0.361 \\
\hline $\begin{array}{l}\text { Nov- } \\
17\end{array}$ & 0.215 & 0.830 & 0.909 & 0.363 & -0.922 & 0.357 & 0.387 & 0.699 \\
\hline $\begin{array}{l}\text { Dec- } \\
17\end{array}$ & 0.830 & 0.406 & -0.253 & 0.800 & 1.184 & 0.237 & 1.254 & 0.210 \\
\hline $\begin{array}{l}\text { Jan- } \\
18\end{array}$ & 0.159 & 0.873 & -1.042 & 0.297 & 1.886 & 0.059 & -0.480 & 0.631 \\
\hline $\begin{array}{l}\text { Feb- } \\
18\end{array}$ & 0.324 & 0.746 & -0.903 & 0.367 & -0.360 & 0.719 & -0.480 & 0.631 \\
\hline $\begin{array}{l}\text { Mar- } \\
18\end{array}$ & 1.412 & 0.158 & 0.274 & 0.784 & -1.062 & 0.288 & -0.480 & 0.631 \\
\hline Apr-18 & -1.944 & 0.052 & -0.441 & 0.659 & -1.904 & 0.057 & -0.480 & 0.631 \\
\hline $\begin{array}{l}\text { May- } \\
18\end{array}$ & -0.837 & 0.402 & 0.600 & 0.548 & 0.201 & 0.841 & -0.047 & 0.963 \\
\hline $\begin{array}{l}\text { Jun- } \\
18\end{array}$ & -1.052 & 0.293 & -0.767 & 0.443 & 1.464 & 0.143 & -0.480 & 0.631 \\
\hline Jul-18 & -1.052 & 0.293 & 0.385 & 0.700 & -0.922 & 0.357 & -0.047 & 0.963 \\
\hline $\begin{array}{l}\text { Aug- } \\
18\end{array}$ & -0.632 & 0.527 & 0.045 & 0.964 & -0.220 & 0.826 & -0.914 & 0.361 \\
\hline $\begin{array}{l}\text { Sep- } \\
18\end{array}$ & 0.377 & 0.706 & 0.808 & 0.419 & -1.624 & 0.104 & -0.047 & 0.963 \\
\hline Oct-18 & 0.046 & 0.963 & 1.008 & 0.313 & -0.220 & 0.826 & -0.914 & 0.361 \\
\hline $\begin{array}{l}\text { Nov- } \\
18\end{array}$ & -0.437 & 0.662 & 1.057 & 0.290 & 1.464 & 0.143 & -0.480 & 0.631 \\
\hline $\begin{array}{l}\text { Dec- } \\
18\end{array}$ & -0.069 & 0.945 & -0.192 & 0.847 & 0.763 & 0.446 & 0.387 & 0.699 \\
\hline $\begin{array}{l}\text { Jan- } \\
19\end{array}$ & -0.373 & 0.709 & -0.378 & 0.706 & 0.763 & 0.446 & 2.554 & 0.011 \\
\hline $\begin{array}{l}\text { Feb- } \\
19\end{array}$ & 0.103 & 0.918 & -0.700 & 0.484 & 0.061 & 0.952 & 0.387 & 0.699 \\
\hline $\begin{array}{l}\text { Mar- } \\
19\end{array}$ & -0.566 & 0.571 & -0.767 & 0.443 & -0.782 & 0.434 & -0.047 & 0.963 \\
\hline Apr-19 & -0.373 & 0.709 & -0.378 & 0.706 & -0.782 & 0.434 & 0.387 & 0.699 \\
\hline $\begin{array}{l}\text { May- } \\
19\end{array}$ & -1.052 & 0.293 & 0.161 & 0.872 & 1.324 & 0.185 & -0.914 & 0.361 \\
\hline $\begin{array}{l}\text { Jun- } \\
19\end{array}$ & -0.908 & 0.364 & -0.835 & 0.404 & 2.166 & 0.030 & -0.047 & 0.963 \\
\hline Jul-19 & -0.700 & 0.484 & 0.859 & 0.391 & -0.782 & 0.434 & -0.047 & 0.963 \\
\hline $\begin{array}{l}\text { Aug- } \\
19\end{array}$ & 0.046 & 0.963 & 0.330 & 0.742 & 0.201 & 0.841 & -0.914 & 0.361 \\
\hline $\begin{array}{l}\text { Sep- } \\
19\end{array}$ & -0.069 & 0.945 & 0.959 & 0.338 & -1.062 & 0.288 & -0.480 & 0.631 \\
\hline
\end{tabular}




\begin{tabular}{|llllllllll|} 
Oct-19 & -0.129 & 0.898 & 1.008 & 0.313 & -1.343 & 0.179 & 1.687 & 0.092 \\
\hline $\begin{array}{l}\text { Nov- } \\
19\end{array}$ & 0.269 & 0.788 & 0.161 & 0.872 & 0.903 & 0.367 & 0.820 & 0.412 \\
$\begin{array}{l}\text { Dec- } \\
19\end{array}$ & -0.011 & 0.991 & 0.439 & 0.660 & 0.763 & 0.446 & 3.421 & 0.001 \\
$\begin{array}{l}\text { Jan- } \\
20\end{array}$ & -0.069 & 0.945 & 1.343 & 0.179 & -0.641 & 0.521 & 0.820 & 0.412 \\
\hline $\begin{array}{l}\text { Feb- } \\
20\end{array}$ & 0.830 & 0.406 & -2.568 & 0.010 & -1.343 & 0.179 & 1.254 & 0.210 \\
$\begin{array}{l}\text { Mar- } \\
20\end{array}$ & 2.773 & 0.006 & -2.477 & 0.013 & 0.763 & 0.446 & -0.914 & 0.361 \\
\hline Apr-20 & 3.611 & $<0.001$ & -2.477 & 0.013 & -0.501 & 0.617 & -0.480 & 0.631 \\
\hline
\end{tabular}

Figures

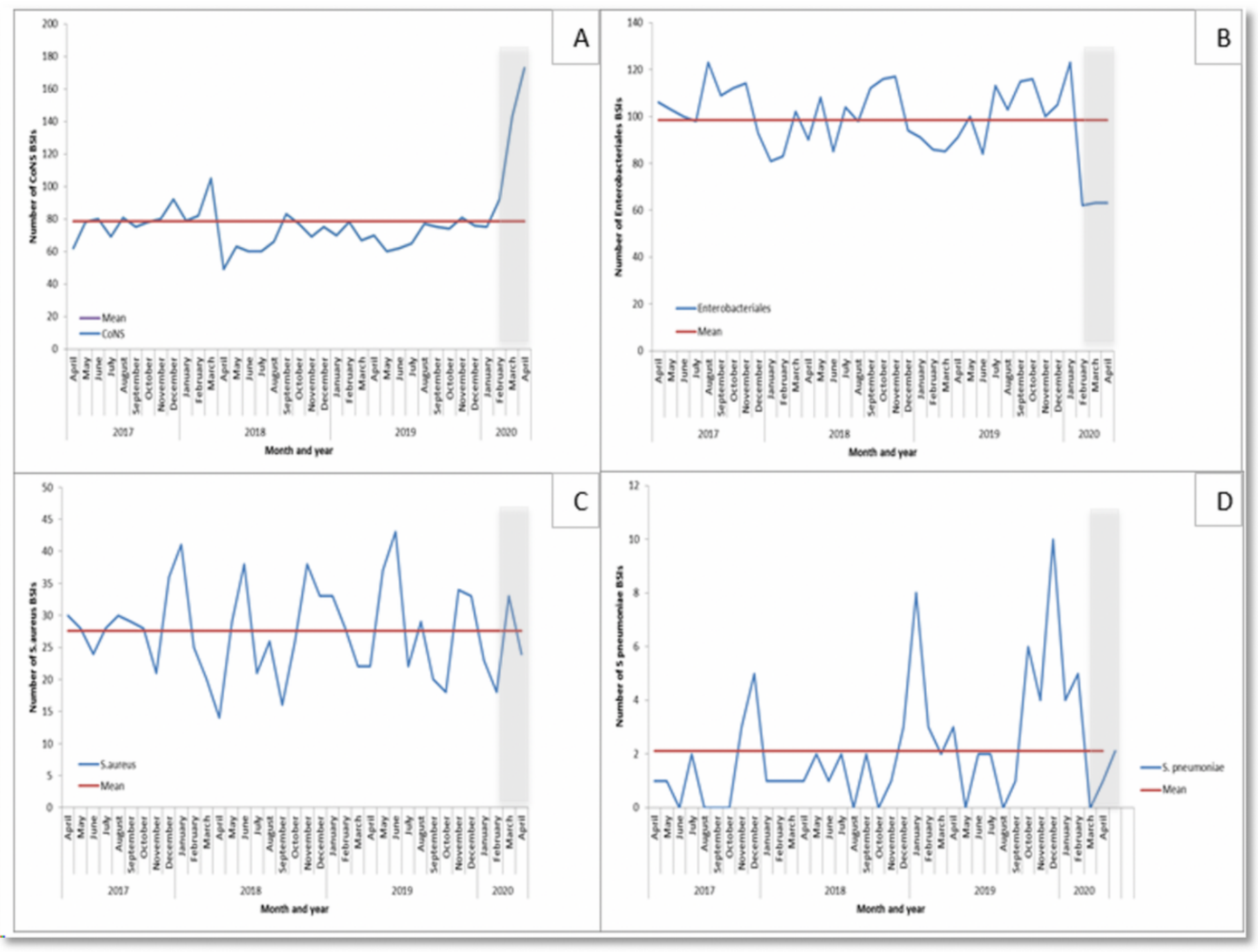

\section{Figure 1}

Comparison of observed blood stream infection with linear trend analysis during the period April 2017 to April 2020 across a six London hospital network for A) coagulase negative staphylococci, B) Enterobacterales, C) S.aureus, and D) S.pneumoniae. 


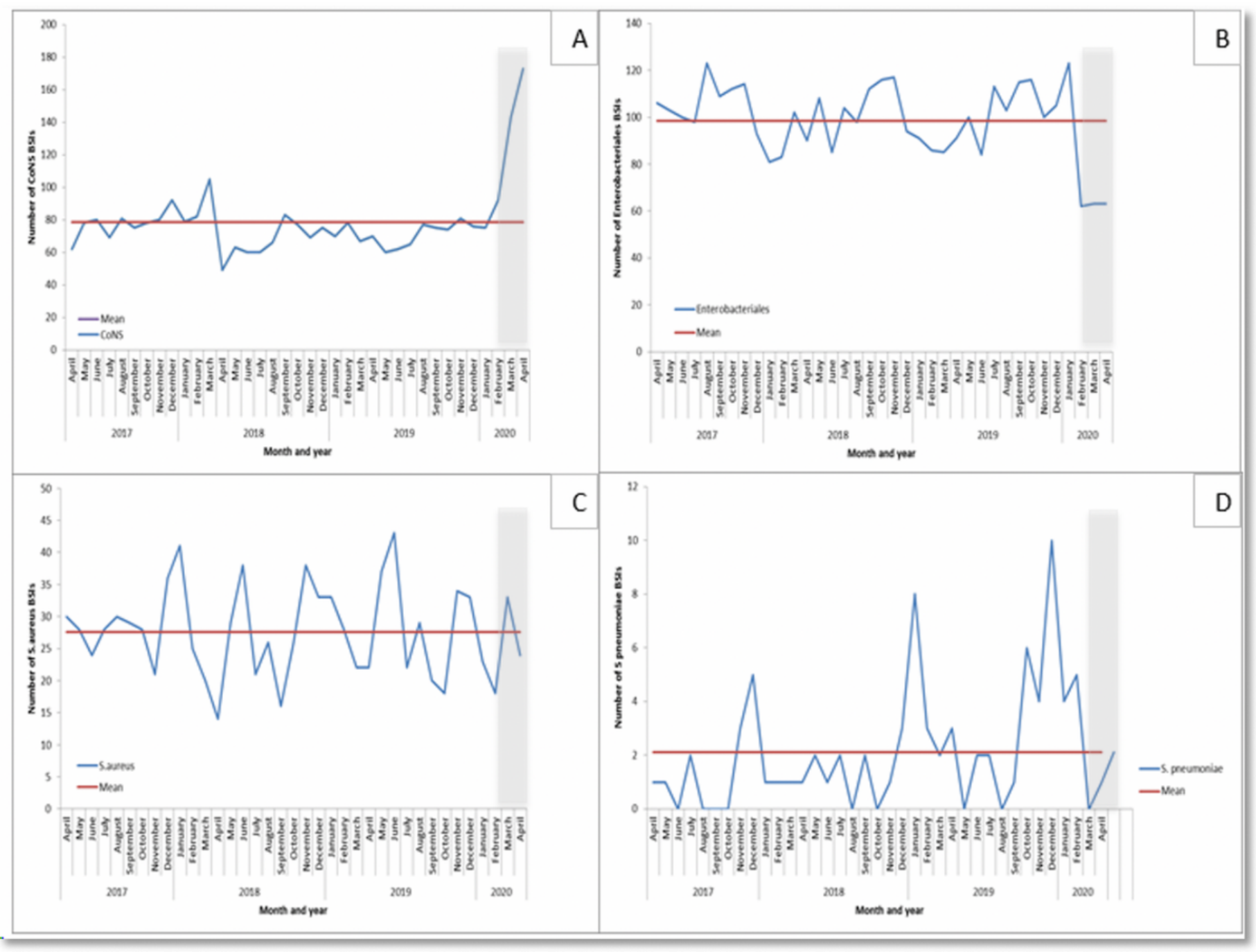

Figure 1

Comparison of observed blood stream infection with linear trend analysis during the period April 2017 to April 2020 across a six London hospital network for A) coagulase negative staphylococci, B) Enterobacterales, C) S.aureus, and D) S.pneumoniae.

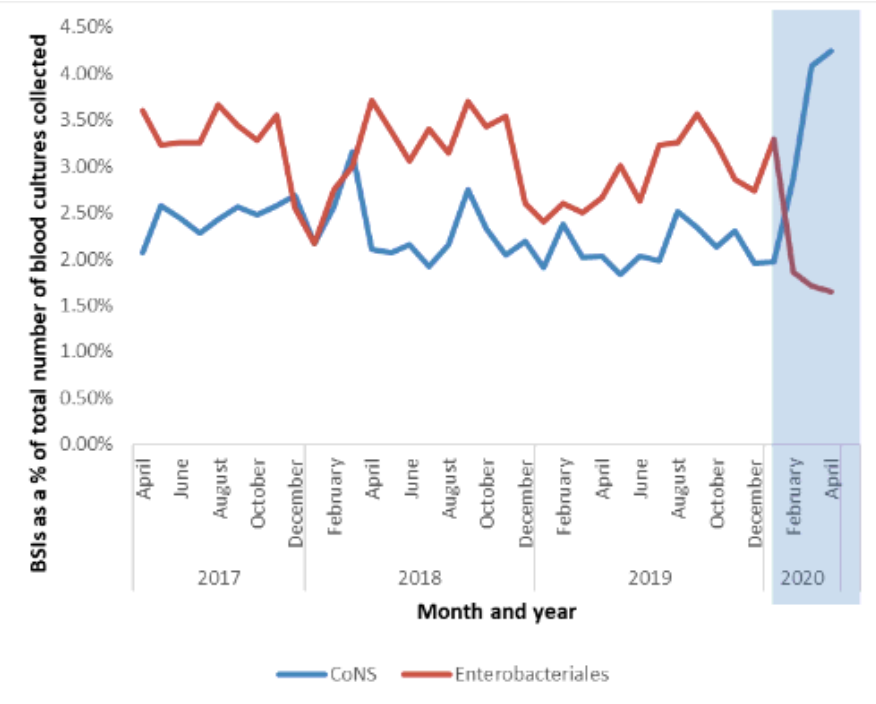

\section{Figure 2}

Change in blood culture isolates across six London hosptials during the COVID-19 social isolation measures in March and April 2020. Enterobacterales and CoNS are shown and expressed as a percentage of the total number of blood cultures collected. 


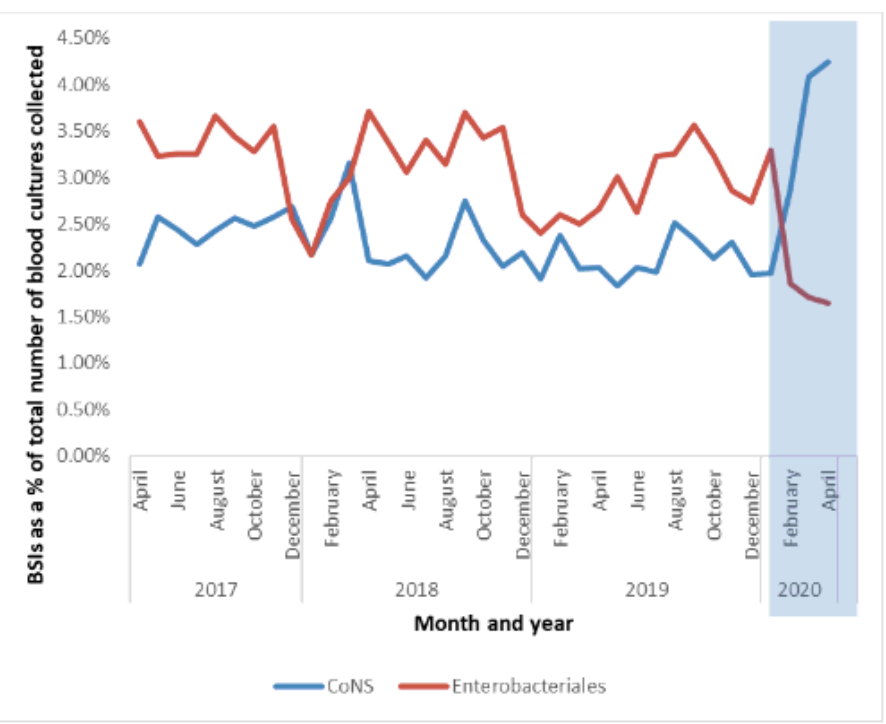

Figure 2

Change in blood culture isolates across six London hosptials during the COVID-19 social isolation measures in March and April 2020. Enterobacterales and CoNS are shown and expressed as a percentage of the total number of blood cultures collected.
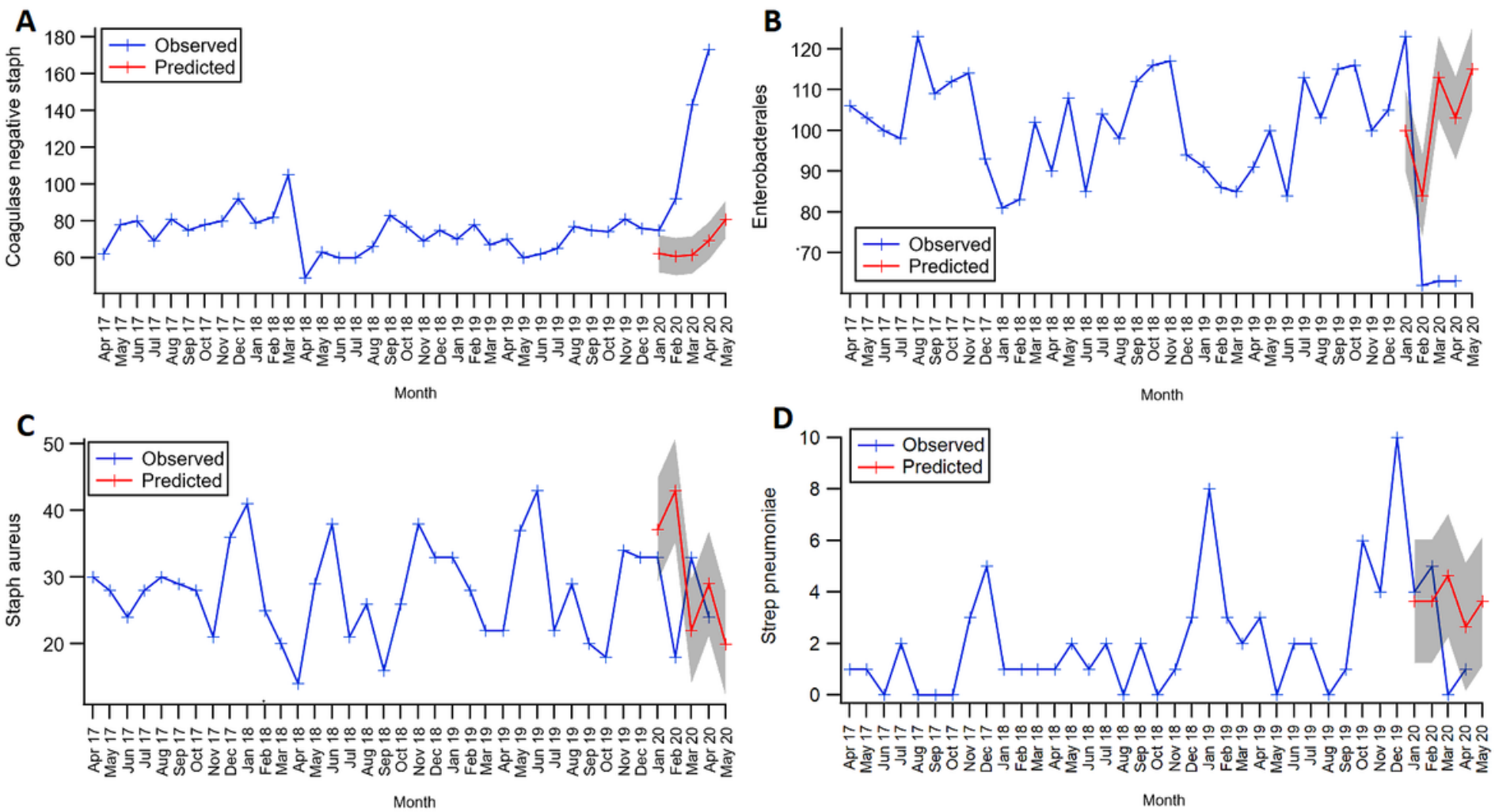

\section{Figure 3}

Comparison of observed versus predicted (ARIMA model Jan-June 2020) trends in blood stream infection during the period April 2017 to April 2020 across a six London hospital network for A) Coagulase negative Staphylococci, B) Enterobacteriales, C) S.aureus, and D) S.pneumoniae. 

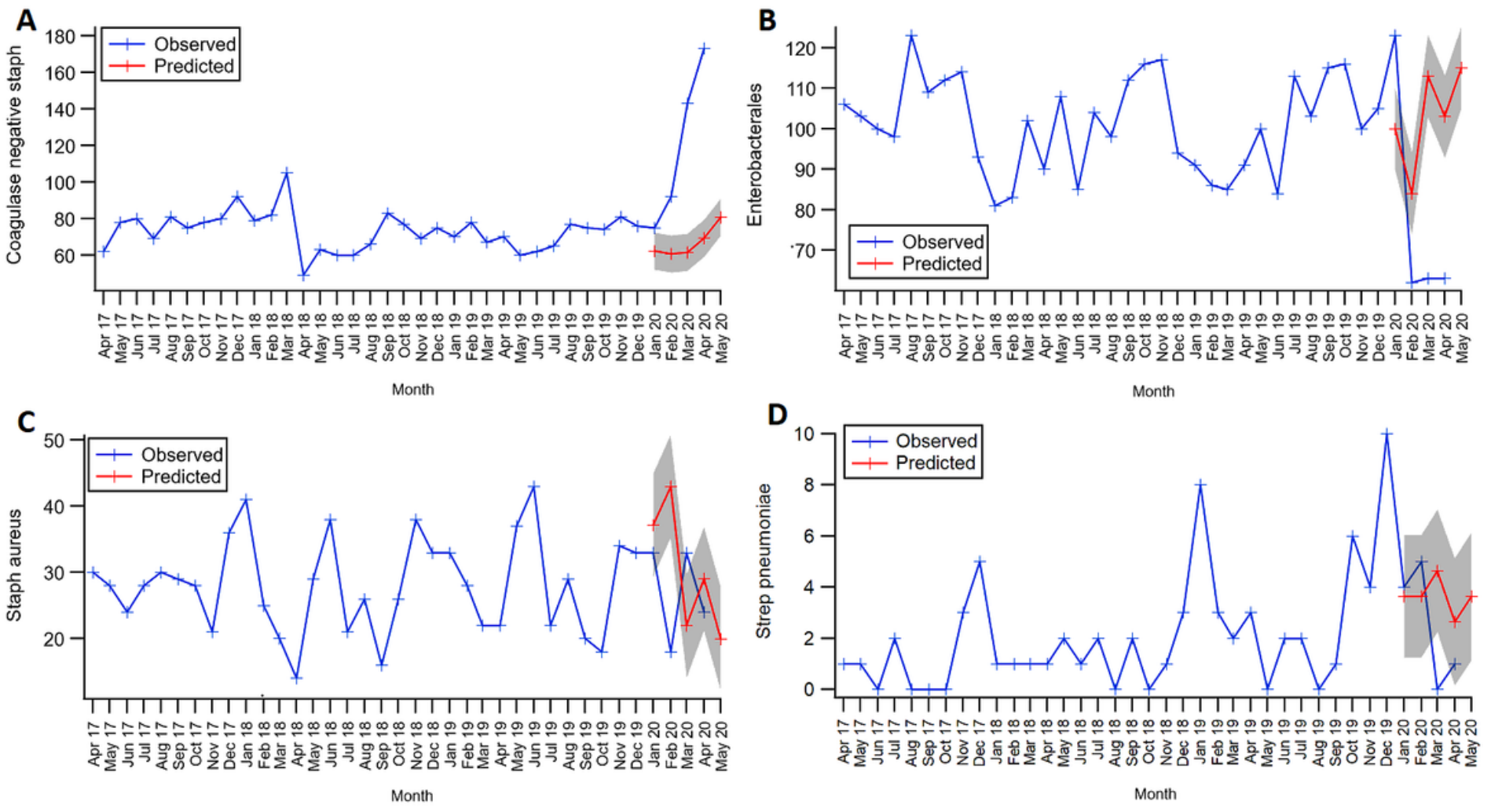

Figure 3

Comparison of observed versus predicted (ARIMA model Jan-June 2020) trends in blood stream infection during the period April 2017 to April 2020 across a six London hospital network for A) Coagulase negative Staphylococci, B) Enterobacteriales, C) S.aureus, and D) S.pneumoniae. 